\title{
Next-generation Sequencing Generated Discrepancy in Abundance Characterization of Complex Microbial Community Compositions: an Error of Bioinformatics Pipeline
}

\author{
Huimin Zhang ${ }^{1}$, Hongkui $\mathrm{He}^{2}$, Runjie $\mathrm{Cao}^{2}$, Huizhi Tang ${ }^{2}$, Zhizhou Zhang ${ }^{1}{ }^{+}$, Anjun $\mathrm{Li}^{2}$ and Jie \\ Jiang $^{1 *}$ \\ ${ }^{1}$ School of Marine Science and Technology, Marine anti-fouling Engineering Technology Center of \\ Shandong Province, Harbin Institute of Technology, Harbin, China 150006 \\ ${ }^{2}$ The Anhui GuJingTribute Liquor Ltd, Bozhou, Anhui, China 236800
}

\begin{abstract}
Next generation sequencing on metagenomes produces a lot of valuable biological and biomedical data but still with some errors. For examples, chimeras are basically originated from biological reactions, while taxonomic classification errors are easily resulted from bioinformatics pipelines. In this study the microbial compositions in the starter (Daqu) of Chinese GujingTribute liquor, especially the dominant species or OTUs (operational taxonomic unit), were determined by two approaches, one is the near full length ribosome gene (16S rDNA plus the internal transcribed spacer (ITS)) library sequencing, and another is $16 \mathrm{~S}$ rDNA V4-V5 region based next generation sequencing approach. The two approaches gave discrepant results for both the prokaryotic microbes and eukaryotic ones. Especially, the results for prokaryotic microbes showed apparent differences in that (1)The most dominant species or OTU belong to different phyla; (2) The 20 most dominant species or OTUs overlapped only partially. Further investigation indicated that the bioinformatics analysis pipeline itself was sometimes an important source for discrepancy generation.
\end{abstract}

Keywords: next generation sequencing, bioinformatics pipeline, metagenome, discrepancy

\section{Introduction}

Progresses in microfluidic machinery and nanotechnology brought tremendous improvement in high throughput biotechnologies, including different platforms of next generation DNA sequencing. Such platforms are both technology pipelines and bioinformatics pipelines. In life sciences and environmental engineering fields, one hot spot is metagenome-based DNA sequencing and related functional studies, in which bioinformatics pipelines employ simple statistical algorithms and a series of DNA databases to classify a large amount of DNA sequences deciphered from thousands of microbial community species. Apparently, the classification accuracy is very important because it may affect a lot on the final biological conclusion or medical detection decision.

There are a batch of typical metagenome sequencing study cases, including soil [1], gut [2], marine water [3], fermentation pits [4], waste treatment microbes [5], sea bed [6], and some specific niche such as hospital hallway air microbes. At present, there are several methods to decode microbial population structures, including traditional pure culture [7], library sequencing [8-9], PCR-DGGE [10], and several types of nextgeneration sequencing (NGS) [11-12]. NGS technology is rapidly employed in hundreds of laboratories with lower and lower cost, but the short read of target genes (such as 16S rDNA variable regions) brings a great limitation on sequencing data implications. NGS technologies using full-length ribosome genes are

\footnotetext{
${ }^{+}$Corresponding author. Zhizhou Zhang Tel.: + 86-631-5683176; fax: +86-631-5687230.
}

E-mail address: zhangzzbiox@hitwh.edu.cn. 
considered still inmature [13] and may need to take several years to be fully developed. Meanwhile, the de novo sequencing technology [14] can assemble long genome fragments for dominant species in a metagenome sample with, unfortunately, still very high cost.

Hahn et al [16] employed two NGS platforms, MiSeq and PacBio RSII, to characterize the cystic fibrosis lung microbiome, and found that MiSeq allowed for the observation of many more operational taxonomic units (OTUs) and higher Chaol and Shannon indices than the PacBio RSII, while only PacBio RSII was able to identify Burkholderia, an important cystic fibrosis pathogen. Such results, if used in supplementary diagnosis, would lead to different decisions because only one platform was able to detect the pathogen.

This study used a complex microbial community sample, the fermentation starter (Daqu), in the ethanol industry. GujingTribute [15] is one of the representative strong-aroma types of Chinese liquor. However, knowledge of the relationship between its flavor and fermentative microbes has been little, so detailed deciphering of Daqu microbial compositions shall be the first step to investigate the above relationship.

In this study, the microbial composition was decoded by two approaches, one is the Illumina Miseq for 16S rDNA V4-V5 plus ITS region (NGS approach), and another is full-length 16S rDNA ITS amplificationTA cloning-Sanger sequencing (TA-clone approach). The two approaches were expected to generate the highly similar namelists of dominant species (or OTUs) for the same Daqu sample, but the results showed that there were apparent discrepancy between the namelists, and especially, the most dominant species in TA-clone approach was not the most dominant OUT in the NGS approach. Further analysis indicated that the error was largely derived from the database quality, a key element in the NGS bioinformatics pipeline.

\section{Materials and Methods}

\subsection{Sampling}

Randomly selected twelve Daqu bricks were smashed and well-mixed into one mix-sample. Metagenome DNA was extracted as previously published protocol [15], then subjected to Illumina Miseq next generation sequencing platform (16S rDNA V4-5) and full-length 16S /ITS rDNA TA-cloning to decipher the microbial compositions.

\subsection{Decoding microbial population composition by next generation sequencing (NGS)}

Metagenomic DNA was extracted from the above prepared production Daqu sample using Solarbio D2600 kit for genome purification. Each 200mg sample generated 100ul metagenome DNA. The 390bp V4V5 region of the $16 \mathrm{~S}$ rRNA gene was amplified using the primer set $520 \mathrm{~F}\left(5^{\prime}-7 \mathrm{bp}\right.$ barcode + GCA CCT AAY TGG GYD TAA AGNG-3') and 904R (5'- CCG TCA ATT CCT TTR AGT TT -3'). For ITS, two primers were use as follow: ITS1 (5'-TCCGTA GGT GAA CCT GCG G-3') and ITS4 (5'-TCC TCC GCT TAT TGA TAT GC-3'). PCR was set up with high fidelity system (0.25ul Q5 high-fidelity DNA polymerase, $5 \mathrm{ul}$ $5 \times$ Reaction Buffer, $5 \mathrm{ul} 5 \times$ High GC Buffer , 0.5ul dNTP $(10 \mathrm{mM}), 1 \mathrm{ul}$ each primer $(10 \mathrm{uM})$, dH2O $11.25 \mathrm{ul})$ and performed according to the following: $98^{\circ} \mathrm{C}-30 \mathrm{~s},\left(98^{\circ} \mathrm{C}-15 \mathrm{~s}, 50^{\circ} \mathrm{C}-30 \mathrm{~s}, 72^{\circ} \mathrm{C}-30 \mathrm{~s}\right)$ for 26 cycles, $72^{\circ} \mathrm{C}$ $30 \mathrm{~s}$ plus $72^{\circ} \mathrm{C}-5 \mathrm{~min}$. PCR amplicons were purified, further processed and subjected to Illumina Miseq platform. All the raw sequence data were processed in the QIIME pipeline [17]. PCR chimeras were checked and removed using the UCHIME software. The remaining good-quality sequences were clustered into operational taxonomic units (OTUs) using a 97\% identity threshold with QIIME's UCLUST tool. The most abundant sequence of each OTU was picked as the representative sequence. The taxonomic information of each representative sequence, also the taxonomic information of each OTU, was annotated using Greengenes database Release 13.8 classifier [18].

\subsection{Deciphering microbial community structures with full-length $16 S$ /ITS rDNA TA- cloning (TA-clone)}

The whole experimental process can be seen in our lab's publication using the universal primers $27 \mathrm{~F}$ and 1492R [15]. For ITS amplification, primer ITS1 (5'-TCCGTA GGT GAA CCT GCG G-3') and ITS4 (5'TCC TCC GCT TAT TGA TAT GC-3') were used. Positive colonies were subjected to Sanger sequencing using the same PCR primers. Each pair of bidirectional sequences was assembled as one single sequence with correct direction. Short sequences without primers on both ends were removed from the data and the 
residual vector bases and primer bases on both ends were deleted (LaserGene, DNAStar). Then the sequences were subjected to chimera checking using UCHIME [19] in mothur software [20]. The sequences with chimeric parts were removed from the data. Finally, the remained sequence was used for further taxonomic analysis. For ITS sequences the UNITE Database was employed using User-friendly Nordic ITS Ectomycorrhiza Database (https://unite.ut.ee/index.php) [21]. The obtained taxonomic information was inspected and corrected manually based on known microbial community knowledge and BLAST (Basic Local Alignment Search Tool) plus RDP (rdp.cme.msu.edu) results. After chimera removal, refined sequences were deposited in the GenBank under the accession numbers KX603403-KX603652 (bacterial 16S rRNA) and KX911990-KX912161 (fungal ITS).

\section{Results and Discussion}

\subsection{Bacterial compositions}

For NGS approach, a total of 30353 good quality prokaryotic sequences with an average length of $390 \mathrm{bp}$ were obtained after quality filtering. At the $97 \%$ identity level, 515 OTUs were classified (data not shown). The most dominant twenty OTUs were listed in Table 1. For TA-clone approach, using a 97\% cutoff, 215 among 249 sequenced clones showed species-level information (data not shown). The most dominant ten species were listed in Table 2. The prefixes "p_", "g_", "s_" indicated OTUs were annotated to the level of phylum, genus, or species, respectively.

Table 1. The most abundant 20 prokaryotic OTUs detected with 16S rDNA V4-5 (OTU level)

\begin{tabular}{|c|c|c|c|c|}
\hline & \#OTU ID & Read & Abundance $(\%)$ & taxonomy \\
\hline 1 & denovo450 & 11970 & 39.43 & p_Proteobacteria; g_Erwinia $;$ s \\
\hline 2 & denovo41 & 2301 & 7.58 & p_Firmicutes; g_Staphylococcus; s_succinus $_{\text {_ }}$ \\
\hline 3 & denovo376 & 1899 & 6.25 & p_Firmicutes; g_Leuconostoc; $\mathrm{s}_{-}$ \\
\hline 4 & denovo105 & 1581 & 5.20 & $\mathrm{p} \_$Actinobacteria; g_Streptomyces; $\mathrm{s}$ \\
\hline 5 & denovo380 & 1511 & 4.97 & p_Firmicutes; g_Lactobacillus; s_paraplantarum \\
\hline 6 & denovo76 & 1434 & 4.72 & p_Firmicutes; g_Lactobacillus; s_brevis \\
\hline 7 & denovo309 & 1267 & 4.17 & p_Firmicutes; g_Thermoactinomyces; $\mathrm{s}$ _sanguinis \\
\hline 8 & denovo379 & 1128 & 3.71 & p_Firmicutes; g_Lactobacillus; $\mathrm{s}$ \\
\hline 9 & denovo420 & 748 & 2.46 & p_Proteobacteria; g_Sarcandra; s_grandifolia \\
\hline 10 & denovo177 & 573 & 1.88 & p_Firmicutes; g_Lactobacillus; $\mathrm{s}$ \\
\hline 11 & denovo99 & 529 & 1.74 & p_Firmicutes; g_Bacillus; $\mathrm{s}$ \\
\hline 12 & denovo178 & 528 & 1.73 & p_Cyanobacteria; $g_{-} ; s_{-}$ \\
\hline 13 & denovo 282 & 478 & 1.57 & p_Proteobacteria; g_Burkholderia; $\mathrm{s}$ \\
\hline 14 & denovo312 & 414 & 1.36 & p_Proteobacteria; g_Enterobacter $; \mathrm{s}$ \\
\hline 15 & denovo220 & 301 & 0.99 & p_Proteobacteria; g_Rhodanobacter; $\mathrm{s}_{-}$ \\
\hline 16 & denovo144 & 221 & 0.72 & p_Proteobacteria; g_Erwinia $;$ s_oleae \\
\hline 17 & denovo6 & 211 & 0.69 & p_Firmicutes; g_Pediococcus; $\mathrm{s}$ _acidilactici \\
\hline 18 & denovo476 & 201 & 0.66 & p_Proteobacteria; g_Pseudomonas; $\mathrm{s}$ \\
\hline 19 & denovo154 & 169 & 0.55 & p_Firmicutes; $\mathrm{g}$ _Lactobacillus $; \mathrm{s}$ \\
\hline 20 & denovo330 & 138 & 0.45 & p_Actinobacteria; g_Rhodococcus; $\mathrm{s}$ \\
\hline
\end{tabular}

Table 2. Bacterial compositions in GujingTribute Daqu determined by TA-clone approach (species level)

\begin{tabular}{|c|c|c|c|c|}
\hline & Species & Abundance (\%) & Taxonomy & $\begin{array}{ll}\text { BLAST } & \text { similarity } \\
\text { level }(\%) & \end{array}$ \\
\hline 1 & Virgibacillus halotolerans & 38.37 & p Firmicutes; $\mathrm{g}$ Virgibacillus & 99 \\
\hline 2 & Thermoactinomyces sanguinis & 19 & p Firmicutes; g Thermoactinomyces & 99 \\
\hline 3 & Virgibacillus $s p$. & 6.48 & p_Firmicutes; g Virgibacillus & 100 \\
\hline 4 & Lactobacillus plantarum & 6.34 & p Firmicutes; g Lactobacillus & 99 \\
\hline 5 & Pantoea agglomerans & 5.94 & p_Proteobacteria; g_Pantoea & 99 \\
\hline 6 & Staphylococcus sp. & 4.52 & p Firmicutes; g Staphylococcus & 100 \\
\hline 7 & Pantoea vagans & 4.32 & p_Proteobacteria; g Pantoea & 99 \\
\hline 8 & Lactobacillus $s p$. & 3.24 & p Firmicutes; g_Lactobacillus & 98 \\
\hline 9 & Bacillus sp. & 2.16 & p_Firmicutes; g_Bacillus & 99 \\
\hline 10 & Staphylococcus kloosii & 2.16 & p_Firmicutes; g_Staphylococcus & 100 \\
\hline 11 & Bacillus subtilis & 1.62 & p_Firmicutes; g_Bacillus & 99 \\
\hline 12 & Bacillus licheniformis & 0.74 & p Firmicutes; g Bacillus & 99 \\
\hline 13 & Planomicrobium sp. & 0.54 & p Firmicutes; g Planomicrobium & 100 \\
\hline 14 & Lactobacillus brevis & 0.54 & p Firmicutes; g_Lactobacillus & 100 \\
\hline 15 & Lactobacillus fermentum & 0.54 & p_Firmicutes; g_Lactobacillus & 99 \\
\hline
\end{tabular}




\begin{tabular}{|c|l|l|l|l|}
\hline 16 & Lactobacillus pontis & 0.54 & $\mathrm{p}$ Firmicutes; $\mathrm{g}$ _Lactobacillus & 99 \\
\hline 17 & Lactobacillus rossiae & 0.54 & $\mathrm{p}$ Firmicutes; g_Lactobacillus & 100 \\
\hline 18 & Weissella sp. & 0.54 & $\mathrm{p}$ Firmicutes; g_Weissella & 100 \\
\hline 19 & Enterobacter hormaechei & 0.54 & p_Proteobacteria; $\mathrm{g}$ Enterobacter & 99 \\
\hline 20 & Pantoea ananatis & 0.54 & p_Proteobacteria; $\mathrm{g}$ Pantoea & 99 \\
\hline
\end{tabular}

Table 3. Bacterial compositions in GujingTribute Daqu (genus level)

\begin{tabular}{|l|l|l|l|}
\hline \multicolumn{2}{|c|}{ TA-clone } & \multicolumn{2}{c|}{ NGS } \\
\hline Abundance (\%) & Taxonomy & Abundance (\%) & Taxonomy \\
\hline 44.85 & p_Firmicutes; g_Virgibacillus; & 40.15 & p_Proteobacteria; g_Erwinia \\
\hline 19 & p_Firmicutes; g_Thermoactinomyces & 15.83 & p_Firmicutes; g_Lactobacillus \\
\hline 11.74 & p_Firmicutes; g_Lactobacillus & 7.58 & p_Firmicutes; g_Staphylococcus \\
\hline 10.8 & p_Proteobacteria; g_Pantoea & 6.25 & p_Firmicutes; g_Leuconostoc \\
\hline 6.68 & p_Firmicutes; g_Staphylococcus & 5.20 & p_Actinobacteria; g_Streptomyces \\
\hline 4.52 & p_Firmicutes; g_Bacillus & 4.17 & p_Firmicutes; g_Thermoactinomyces \\
\hline 0.54 & p_Firmicutes; g_Planomicrobium & 2.46 & p_Proteobacteria; g_Sarcandra \\
\hline 0.54 & p_Firmicutes; g_Weissella & 1.74 & p_Firmicutes; g_Bacillus \\
\hline 0.54 & p_Proteobacteria; g_Enterobacter & 1.73 & p_Cyanobacteria; g_ \\
\hline
\end{tabular}

\subsection{Eukaryotes compositions}

For eukaryotic data, a total of 15591 good quality sequences with an average length of 265 bp were obtained after quality filtering. At the $97 \%$ identity level, 84 OTUs were classified (data not shown). The most dominant twenty OTUs were listed in Table 4. For TA-clone approach, all 172 curated sequences of ITS clones can give species-level annotation (data not shown). The most dominant ten species were listed in Table 5 .

Table 4. The most abundant 20 eukaryotic OTUs detected with ITS (OTU level)

\begin{tabular}{|c|c|c|c|c|}
\hline & \#OTU ID & Read & Abundance (\%) & taxonomy \\
\hline 1 & denovo61 & 4623 & 29.65 & p_Ascomycota; g_Aspergillus; s_Aspergillus flavus \\
\hline 2 & denovo60 & 2903 & 18.61 & p_Zygomycota; g_Rhizopus; s_Rhizopus arrhizus \\
\hline 3 & denovo46 & 2627 & 16.84 & p_Zygomycota; g_Rhizomucor; s_Rhizomucor pusillus \\
\hline 4 & denovo66 & 791 & 5.07 & p_Ascomycota; g_Paecilomyces; s_Paecilomyces verrucosus \\
\hline 5 & denovo42 & 754 & 4.83 & p_Ascomycota; g_unidentified; s_Saccharomycetales $s p$ \\
\hline 6 & denovo43 & 661 & 4.23 & p_Ascomycota; g_Aspergillus; s_Aspergillus cibarius \\
\hline 7 & denovo19 & 580 & 3.72 & p_unidentified; g_unidentified; s_Plantae $s p$ \\
\hline 8 & denovo21 & 519 & 3.32 & p_Ascomycota; g_Thermomyces; s_Thermomyces lanuginosus \\
\hline 9 & denovo6 & 488 & 3.13 & p_Ascomycota; g_Candida; $\mathbf{s \_ C C a n d i d a}$ xylopsoci \\
\hline & denovo49 & 407 & 2.61 & p_unidentified; g_unidentified; $s \_$Plantae $s p$ \\
\hline & denovo64 & 232 & 1.48 & p_Ascomycota; g_unidentified; s_Eurotiomycetes $s p$ \\
\hline 12 & denovo4 & 159 & 1.01 & p_Zygomycota; g_Lichtheimia; s_Lichtheimia ornata \\
\hline 13 & denovo51 & 116 & 0.74 & p_Ascomycota; g_Thermoascus; s_Thermoascus aurantiacus \\
\hline 14 & denovo 28 & 108 & 0.69 & p_Ascomycota; g_Aspergillus; $\mathrm{s} \_$Aspergillus piperis \\
\hline 15 & denovo8 & 103 & 0.66 & p_Ascomycota; g_Candida; $\mathrm{s} \_$Candida blattae \\
\hline 16 & denovo5 & 86 & 0.55 & p_Ascomycota; g_Aspergillus; $\mathrm{s}$ _Aspergillus candidus \\
\hline 17 & denovo53 & 60 & 0.38 & p_Ascomycota; g_Wickerhamomyces; s_Wickerhamomyces anomalus \\
\hline 18 & denovo59 & 48 & 0.30 & p_Ascomycota; g_Penicillium; $\mathrm{s}$ _Penicillium polonicum \\
\hline 19 & denovo77 & 38 & 0.24 & p_Ascomycota; $g$ _Penicillium; $\mathrm{s}$ _Penicillium citrinum \\
\hline 20 & denovo68 & 27 & 0.17 & p_Ascomycota; g_Monascus; s_Monascus purpureus \\
\hline
\end{tabular}

Table 5. Eukaryotic compositions in GujingTribute Daqu determined by TA-clone approach (species level)

\begin{tabular}{|c|c|c|c|c|}
\hline & Species & Abundance $(\%)$ & Taxonomy & BLAST similarity level (\%) \\
\hline 1 & Rhizopus_arrhizus & 45.09 & p_Zygomycota;g_Rhizopus & 100 \\
\hline 2 & Aspergillus_flavus & 14.63 & p_Ascomycota; g_Aspergillus & 100 \\
\hline 3 & Thermomyces_lanuginosus & 13.94 & p_Ascomycota; g_Thermomyces & 100 \\
\hline 4 & Aspergillus_amstelodami & 8.02 & p_Ascomycota; g_Aspergillus & 100 \\
\hline 5 & Thermoascus_crustaceus & 6.44 & p_Ascomycota; g_Thermoascus & 99 \\
\hline 6 & Thermoascus_aurantiacus & 5.31 & p_Ascomycota; g_Thermoascus & 100 \\
\hline 7 & Penicillium_chrysogenum & 1.94 & p_Ascomycota; g_Penicillium & 100 \\
\hline 8 & Pichia_kudriavzevii & 1.94 & p_Ascomycota; g_Pichia & 99 \\
\hline 9 & Aspergillus_candidus & 0.96 & p_Ascomycota; g_Aspergillus & 99 \\
\hline 10 & Lichtheimia_ramosa & 0.96 & p_Zygomycota; g_Lichtheimia & 100 \\
\hline
\end{tabular}

\subsection{The discrepancy is related with data processing pipelines}


Though Table 6 suggested that the two approaches generate apparent discrepancy on characterization of eukaryotic species, from the Table 2 and Table 3, however, discrepancy in prokaryotic microbial compositions may be more dramatic. It was clear that the most dominant bacterial species was a Virgibacillus (Firmicute) strain, while Table 1 and Table 3 demonstrated that the most dominant OTU was a Erwinia (Proteobacteria) microbe. Because they belonged to different phyla, the classification discrepancy looked unacceptable. The NGS platform in this study used GreenGenes database to annotate each filtered sequence while the TA-clone approach used BLAST and RDP as annotation tools, and that may be the reason why the most dominant OTU (denovo 450) [22] was classified as an Erwinia species (OTU). If the representative sequence of OTU 450 was tested on BLAST and RDP databases, it would be assigned as a Pantoea agglomerans strain, relatively consistent with the TA-clone result (Table 2) in which Pantoea agglomerans was the $5^{\text {th }}$ most dominant species.

Table 6. Eukaryotic compositions in GujingTribute Daqu (genus level)

\begin{tabular}{|l|l|l|l|}
\hline \multicolumn{2}{|c|}{ TA-clone } & \multicolumn{2}{c|}{ NGS } \\
\hline Abundance (\%) & Taxonomy & Abundance (\%) & Taxonomy \\
\hline 45.09 & p_Zygomycota;g_Rhizopus & 35.12 & p_Ascomycota; g_Aspergillus \\
\hline 23.61 & p_Ascomycota; g_Aspergillus & 18.61 & p_Zygomycota; g_Rhizopus \\
\hline 13.94 & p_Ascomycota; g_Thermomyces & 16.84 & p_Zygomycota; g_Rhizomucor \\
\hline 11.75 & p_Ascomycota; g_Thermoascus & 5.07 & p_Ascomycota; g_Paecilomyces \\
\hline 1.94 & p_Ascomycota; g_Penicillium & 4.83 & p_Ascomycota; g_unidentified \\
\hline 1.94 & p_Ascomycota; g_Pichia & 3.72 & p_unidentified; g_unidentified \\
\hline 0.96 & p_Zygomycota; g_Lichtheimia & 3.32 & p_Ascomycota; g_Thermomyces \\
\hline
\end{tabular}

Metagenome sequencing and sequence annotation rely heavily on the bioinformatics databases in which RDP [rdp.cme.msu.edu], GreenGenes [greengenes.secondgenome.com], SILVA [http://www.arb-silva.de] and NCBI [www.ncbi.nlm.nih.gov/nucleotide] are all good ones. However, for ribosome genes, GreenGenes contains most high-quality sequences and loses the general coverage range in that some different taxonomic elements are only in those low or intermediate quality sequences. For NCBI source, it has many misleading or wrong sequences (such as some chimeras) due to a historic reason and those low quality or wrong sequences have not been re-confirmed and cleaned. However, RDP has a general coverage that is between GreenGenes and NCBI in the context of ribosome gene representations. So as one important step in the bioinformatics pipeline, database quality curation is a continuous and stringent task from now on. On the other hand, choice of a specific NGS protocol for a specified research task is also critical; for example, Yang et al [23] conducted a survey for sensitivity and correlation of hypervariable regions in 16S rRNA genes in phylogenetic analysis and found that V4-V6 region was best to represent the optimal sub-regions of a new phylum.

\section{Conclusion}

In conclusion, metagenome sequencing generates large amount of DNA sequences that are subjected to possible wrong or misleading annotations due to some systematic error in bioinformatics analysis pipelines. Sometimes such an error or discrepancy may lead to big difference in the final biological research conclusion or biomedical detection decision. The results in this study further confirmed that several different approaches shall be considered at the same time for accurately determining compositions of a complex microbial community and database quality curation shall be a systematic and continuous endeavor worldwide. In the future, the authors would like to develop some novel nanoparticle-assisted DNA amplification techniques with higher fidelity than those used in the present NGS approaches so that some sequence errors can be avoided before they enter the following bioinformatics pipelines.

\section{Acknowledgements}

This study was supported by NSFC (No.31071170), GujingTribute fund (2016-1), GREDBIO (201401) and HIT fund (hitwh200904, 2016GSF115022).

\section{References}

[1] Erick C. et al. Forest harvesting reduces the soil metagenomic potential for biomass decomposition. ISME J 2015, 9(11): $2465-2476$. 
[2] Zeng, B. et al. The bacterial communities associated with fecal types and body weight of rex rabbits. Sci Rep 2015, 5,9342 .

[3] Shinichi, S. et al. Structure and function of the global ocean microbiome. Science 2015, 348(6237), 1261359.

[4] Luo, X.M. et al. Phylum-specific primer design and implication in quantification of the microbial community structure in GuJingGong pit mud., Adv Mater Res 2014, 1051,311-316.

[5] Shu D. et al. Metagenomic and quantitative insights into microbial communities and functional genes of nitrogen and iron cycling in twelve wastewater treatment systems. Chem Eng J 2016,290,21-30.

[6] Christner, B.C. et al.A microbial ecosystem beneath the West Antarctic ice sheet. Nature 2014, 512(7514):310-313

[7] Kaeberlein, T., Lewis, K. and Epstein, S.S. Isolating" uncultivable" microorganisms in pure culture in a simulated natural environment. Science 2002, 296, 1127-1129.

[8] Rondon, M.R. et al. Cloning the soil metagenome: a strategy for accessing the genetic and functional diversity of uncultured microorganisms. Appl Environ Micro 2000, 66, 2541-2547.

[9] Gou, M. et al. Characterization of the microbial community in three types of fermentation starters used for Chinese liquor production. J Inst Brew 2015, 121, 620-627.

[10] Zhang, L. et al. Characterisation of microbial communities in Chinese liquor fermentation starters Daqu using nested PCR-DGGE. World J Microbiol Biotechnol 2014, 30, 3055-3063.

[11] Klindworth, A. et al. Evaluation of general 16S ribosomal RNA gene PCR primers for classical and nextgeneration sequencing-based diversity studies. Nucleic Acids Res 2012, gks808.

[12] Hong, X.et al. Metagenomic sequencing reveals the relationship between microbiota composition and quality of Chinese Rice Wine. Sci Rep 2016, 6, 26621

[13] Wagner, J. et al. Evaluation of PacBio sequencing for full-length bacterial 16S rRNA gene classification. BMC Microbiol 2016, 16(1):274.

[14] Hess, M. et al. Metagenomic discovery of biomass-degrading genes and genomes from cow rumen. Science 2011, 331, 463-467.

[15] Zhang, H. et al. Employment of near full-length ribosome gene TA-cloning and Primer-Blast to detect multiple species in a natural complex microbial community using species-specific primers designed with their genome sequences. Mol Biotechnol 2016, 58, 729-737.

[16] Hahn A et al. Different next generation sequencing platforms produce different microbial profiles and diversity in cystic fibrosis sputum. J Microbiol Methods 2016, 130, 95-99.

[17] Navas-Molina, J.A. et al. Advancing our understanding of the human microbiome using QIIME. Methods Enzymol 2013, 531, 371-444.

[18] DeSantis, T.Z. et al. Greengenes, a chimera-checked 16S rRNA gene database and workbench compatible with ARB. Appl Environ Microb 2006, 72, 5069-5072.

[19] Edgar, R.C. et al. UCHIME improves sensitivity and speed of chimera detection. Bioinformatics 2011, 27, 21942200 .

[20] Schloss, P.D. et al. Introducing mothur: open-source, platform-independent, community-supported software for describing and comparing microbial communities. Appl Environ Micro 2009, 75, 7537-7541.

[21] Abarenkov, K. et al. The UNITE database for molecular identification of fungi - recent updates and future perspectives. New Phyto 2010, 186, 281-285.

[22] >OTU 450

TACGGAGGGTGCAAGCGTTAATCGGAATTACTGGGCGTAAAGCGCACGCAGGCGGTCTGTTAAGTCAGATGTGAAATCCCCGGGCTTAA CCTGGGAACTGCATTTGAAACTGGCAGGCTTGAGTCTTGTAGAGGGGGGTAGAATTCCAGGTGTAGCGGTGAAATGCGTAGAGATCTGG AGGAATACCGGTGGCGAAGGCGGCCCCCTGGACAAAGACTGACGCTCAGGTGCGAAAGCGTGGGGAGCAAACAGGATTAGATACCCTG GTAGTCCACGCCGTAAACGATGTCGACTTGGAGGTTGTTCCCTTGAGGAGTGGCTTCCGGAGCTAACGCGTTAAGTCGACCGCCTGGGGA GTACGGCCGCAAGGTTAAAACTTAAATGAATTGACGG

[23] Yang, B. et al. Sensitivity and correlation of hypervariable regions in 16S rRNA genes in phylogenetic analysis. BMC Bioinformatics 2016, 17, 135. 